\title{
Optical cooling of Nd-doped solids
}

\author{
Angel J. Garcia-Adeva ${ }^{\mathrm{a}}$, Rolindes Balda ${ }^{\mathrm{a}, \mathrm{b}}$, Mohammed Al Saleh ${ }^{\mathrm{a}}$, Sara Garcia-Revilla, ${ }^{\mathrm{a}}$ \\ Daniel Sola ${ }^{\mathrm{b}}$, and Joaquin Fernandez ${ }^{\mathrm{a}, \mathrm{b}}$ \\ ${ }^{a}$ Departamento Física Aplicada I, Escuela Técnica Superior de Ingeniería, Alda. \\ Urquijo s/n 48013 Bilbao, Spain \\ ${ }^{b}$ Centro de Física de Materiales CSIC-UPV/EHU and Donostia International Physics \\ Center, Apartado 1072, 20080 San Sebastián, Spain
}

\begin{abstract}
In this work we present a comprehensive review of recent work carried out by our group in the field of optical refrigeration of Nd-doped solids. Several infrared thermography measurements in Nd-doped $\mathrm{KPb}_{2} \mathrm{Cl}_{5}$ crystals and micro-powders both above and below the barycentre of the ${ }^{4} \mathrm{~F}_{3 / 2}$ are presented. These include some of our most recent ones obtained by employing a novel technique that allows one to perform differential temperature measurements. The role of both the direct anti-Stokes absorption processes and those assisted by either excited state absorption or energy transfer upconversion in the cooling process is discussed.
\end{abstract}

Keywords: Laser cooling, Neodymium, optical properties

\section{INTRODUCTION}

Solid-state cooling by anti-Stokes emission first demonstrated by Epstein [1] and coworkers in 1995 in an ytterbium-doped heavy-metal fluoride glass promoted huge efforts by the scientific community to improve this process and to develop other different materials doped with rare-earths ions. However, in spite of the important results obtained by the Epstein group and other researchers, only a reduced number of $\mathrm{Yb}^{3+}$-doped glasses and crystal matrices [2] were able to produce net cooling and among rare-earth ions only $\mathrm{Tm}^{3+}$-doped heavy-metal fluoride glass and crystals and an $\mathrm{Er}^{3+}$-doped $\mathrm{KPb}_{2} \mathrm{Cl}_{5}$ crystal and a fluorochloride glass were reported to be efficient coolers $[3,4]$.

A few years ago, some of the present authors demonstrated that the efficient infrared-to-visible upconversion present in $\mathrm{Nd}^{3+}$-doped $\mathrm{KPb}_{2} \mathrm{Cl}_{5}$ crystal and powder can lead to local internal and bulk optical cooling [5], in agreement with a previous theoretical model [6]. These results show that new cooling channels based on upconversion processes can be of paramount importance in the search of new rare-earth-doped materials for optical cooling. Moreover, we recently reported a new technique, termed differential infrared thermography (DIT), that overcomes some of the limitations previously found in the standard infrared thermography setup, especially when the optical cooling channel competes with a strong background heating absorption that is present even in undoped materials.

In the present work, we present a comprehensive review of some of the main results obtained along the lines of the previous paragraph. In particular, we pay especial emphasis to the spectral region just above the barycenter of the ${ }^{4} \mathrm{~F}_{3 / 2}$ emiting band, where infrared to visible upconversion is the main contribution to the optical cooling seen in these low-phonon-energy materials, and the mechanisms responsible of optical cooling are briefly described. Also, results of the aforementioned DIT technique in the spectral region below the barycentre of the emitting band are presented and discussed in terms of two-phonon-assisted anti-Stokes processes.

Laser Refrigeration of Solids V, edited by Richard I. Epstein, Mansoor Sheik-Bahae,

Proc. of SPIE Vol. 8275, 827502 - (C) 2012 SPIE · CCC code: 0277-786X/12/\$18 · doi: 10.1117/12.907837 


\section{EXPERIMENTAL}

\subsection{Samples}

Single crystals of non-hygroscopic ternary potassium-lead chloride $\mathrm{KPb}_{2} \mathrm{Cl}_{5}$ doped with $\mathrm{Nd}^{3+}$ ions, typically $2 \mathrm{~cm}$ long and $1 \mathrm{~cm}$ in diameter, have been grown in our laboratory by the Bridgman technique, in a chlorine atmosphere with a two-zone transparent furnace, a vertical temperature gradient of 18 ${ }^{\circ} \mathrm{C} / \mathrm{cm}$, and a $1 \mathrm{~mm}$ per hour growth rate. Quartz ampoules with a pointed end were used as seed selectors to promote single crystal growth. The $\mathrm{Nd}^{3+}$ content was $1 \mathrm{~mol} \%$ in the melt. The plates with approximate dimensions of $4.5 \times 6.5 \times 2.7 \mathrm{~mm}^{3}$ were cut from blocks and polished for spectroscopic measurements.

In order to obtain ground powder out of $\mathrm{KPb}_{2} \mathrm{Cl}_{5}: \mathrm{Nd}^{3+}$ crystals, a (Retsch MM200) mixer mill was employed during $4 \mathrm{~min}$. The polydispersity of the measured powder was evaluated from Scanning Electron Microscopy. The particle size was estimated from the average between the major and minor axis lengths of the grains. The histogram of the particle size was fitted to a log-normal function and an average powder size of $1.2 \pm 0.6 \mu \mathrm{m}$ was obtained. The resulting powder was compacted in a cylindrical quartz cell for handling ease and cooling measurements. The volume filling factor of the powder material $(f=$ 0.55 ) was calculated by measuring sample volume and weight.

\subsection{Spectroscopic measurements}

All the spectroscopic data were taken at room temperature (RT). The absorption spectrum was performed by using a Cary 5 spectrophotometer. The steady-state emission and excitation spectra in the near infrared region were recorded by exciting the sample with a cw Argon ion pumped Ti:sapphire ring laser $\left(0.4 \mathrm{~cm}^{-1}\right.$ linewidth). The luminescence was collected by using a $0.25 \mathrm{~m}$ monochromator and detected by a Hamamatsu R7102 extended IR photomultiplier and finally amplified by a lock-in technique. The upconversion spectra were recorded by exciting the sample with a cw Argon ion pumped Ti:Sapphire ring laser $\left(0.4 \mathrm{~cm}^{-1}\right.$ linewidth). The luminescence was analyzed using a $0.25 \mathrm{~m}$ monochromator and detected by a Hamamatsu R928 photomultiplier and finally amplified by a lock-in technique.

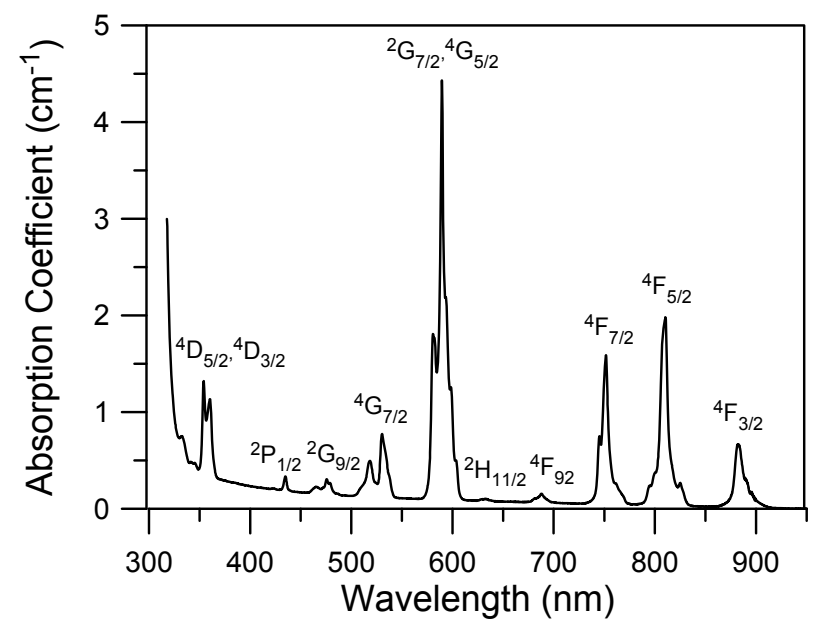

Fig. 1. Room temperature absorption spectrum of $\mathrm{Nd}^{3+}$ ions in $\mathrm{KPb}_{2} \mathrm{Cl}_{5}$ crystal.

\section{OPTICAL PROPERTIES OF Nd ${ }^{3+}$ IN $\mathrm{KPb}_{2} \mathrm{Cl}_{5}$ CRYSTAL}

The room temperature absorption spectrum of $\mathrm{KPb}_{2} \mathrm{Cl}_{5}: \mathrm{Nd}^{3+}$ unoriented crystal in the $300-950 \mathrm{~nm}$ range is shown in Fig. 1. The spectrum shows the typical absorption bands corresponding to the transitions from 
the ${ }^{4} \mathrm{I}_{9 / 2}$ ground state to the excited states of $\mathrm{Nd}^{3+}$ ions.

The steady state emission spectrum obtained under excitation at the high energy side of the ${ }^{4} \mathrm{I}_{9 / 2} \rightarrow{ }^{4} \mathrm{~F}_{5 / 2}$ absorption band at $795 \mathrm{~nm}$ with a Ti-sapphire laser is displayed in Fig. 2. This spectrum shows the emission bands corresponding to transitions ${ }^{4} \mathrm{~F}_{5 / 2} \rightarrow{ }^{4} \mathrm{I}_{9 / 2,11 / 2}$ and ${ }^{4} \mathrm{~F}_{3 / 2} \rightarrow{ }^{4} \mathrm{I}_{9 / 2,11 / 2}$. The presence of the emissions originated from level ${ }^{4} \mathrm{~F}_{5 / 2}$ level is a consequence of the reduced nonradiative multiphonon decay rate of this crystal compared with fluorides and oxides. In oxide and fluoride systems, excitation into the ${ }^{4} \mathrm{~F}_{5 / 2}$ level is followed by fast nonradiative decay to the ${ }^{4} \mathrm{~F}_{3 / 2}$ level due to the relatively high phonon energies. However in this chloride crystal the energy gap between the ${ }^{4} \mathrm{~F}_{5 / 2}$ and ${ }^{4} \mathrm{~F}_{3 / 2}$ levels is around $1046 \mathrm{~cm}^{-1}$ and the energy of the phonons involved $203 \mathrm{~cm}^{-1}$, therefore the ${ }^{4} \mathrm{~F}_{5 / 2}$ level can emit radiatively.

The excitation spectrum of the ${ }^{4} \mathrm{I}_{9 / 2} \rightarrow{ }^{4} \mathrm{~F}_{5 / 2},{ }^{4} \mathrm{~F}_{3 / 2}$ transitions obtained by collecting the luminescence at the maximum of the ${ }^{4} \mathrm{~F}_{3 / 2} \rightarrow{ }^{4} \mathrm{I}_{11 / 2}$ transition is shown in Fig. 3. The spectrum is similar to the absorption spectrum in this region.

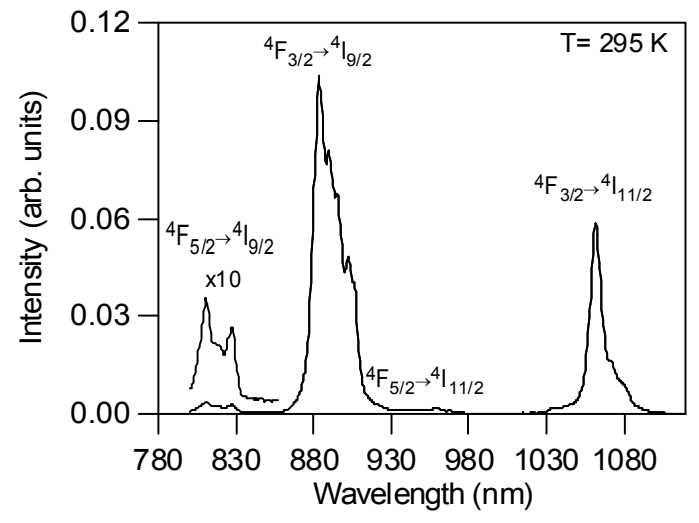

Fig. 2. Steady-state emission spectrum.

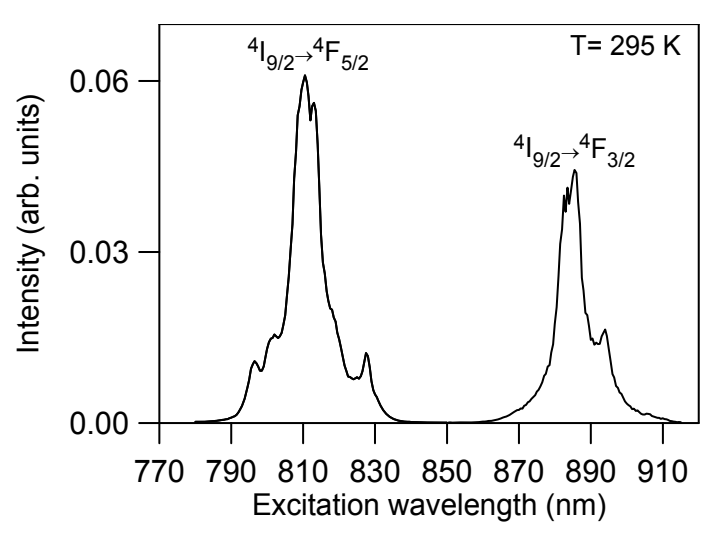

Fig. 3. Excitation spectrum obtained by collecting the luminescence at $1064 \mathrm{~nm}$.

We have observed IR to VIS and UV upconverted emissions at RT when pumping the sample into both the ${ }^{4} \mathrm{~F}_{3 / 2}$ and ${ }^{4} \mathrm{~F}_{5 / 2}$ levels of $\mathrm{Nd}^{3+}$. Figure 4 shows the upconverted emission spectra obtained by exciting the sample at different emission wavelengths between the ${ }^{4} \mathrm{~F}_{3 / 2}$ and ${ }^{4} \mathrm{~F}_{5 / 2}$ levels and at $885 \mathrm{~nm}$, in resonance with the ${ }^{4} \mathrm{I}_{9 / 2} \rightarrow{ }^{4} \mathrm{~F}_{3 / 2}$ transition. As can be seen, the relative intensity of the upconverted emission is different depending on the excitation wavelength. The emission spectrum obtained under excitation at $885 \mathrm{~nm}$ shows three intense emissions in the green, orange, and red regions of the spectrum, located around 535, 595, and $668 \mathrm{~nm}$ respectively. These bands present similar decay rates, indicating that they mainly originate in the same level or group of levels. The energy level diagram and the emission spectra obtained under one photon excitation indicate that the green, orange, and red upconversion emissions correspond to transitions from levels ${ }^{4} \mathrm{G}_{9 / 2}-{ }^{4} \mathrm{G}_{7 / 2}$ to levels ${ }^{4} \mathrm{I}_{9 / 2},{ }^{4} \mathrm{I}_{11 / 2}$ and ${ }^{4} \mathrm{I}_{13 / 2}$ respectively, although the orange and red bands could also contain some contribution from emissions coming from level ${ }^{4} \mathrm{G}_{5 / 2}$. The upconversion spectrum also shows blue peaks located around 420 and $435 \mathrm{~nm}$ and UV emissions located around 362 and $388 \mathrm{~nm}$. Concerning the UV and blue upconverted emissions, the band at $435 \mathrm{~nm}$ corresponds to the ${ }^{2} \mathrm{P}_{1 / 2} \rightarrow{ }^{4} \mathrm{I}_{9 / 2}$ transition. Emissions at 362,388, and $420 \mathrm{~nm}$ have been assigned to transitions from ${ }^{4} \mathrm{D}_{3 / 2}$ state to ${ }^{4} \mathrm{I}_{9 / 2},{ }^{4} \mathrm{I}_{11 / 2}$, and ${ }^{4} \mathrm{I}_{13 / 2}$ levels respectively. Finally, the band located around $760 \mathrm{~nm}$ has been assigned to the ${ }^{4} \mathrm{~F}_{7 / 2} \rightarrow{ }^{4} \mathrm{I}_{9 / 2}$ transition. When pumping the sample into the ${ }^{4} \mathrm{~F}_{5 / 2}$ band of $\mathrm{Nd}^{3+}$, a similar spectrum is obtained. It is worthy to mention that, for all excitations, the visible luminescence is very intense and the sample whole emission looks white with naked eye. In the case of 
the emission spectra obtained by exciting at wavelengths between ${ }^{4} \mathrm{~F}_{3 / 2}$ and ${ }^{4} \mathrm{~F}_{5 / 2}$ levels the most intense emission is the blue peak corresponding to the ${ }^{2} \mathrm{P}_{1 / 2} \rightarrow{ }^{4} \mathrm{I}_{9 / 2}$ transition.

The excitation spectra recorded by monitoring the emissions at 435, $595 \mathrm{~nm}, 760 \mathrm{~nm}$, and $1064 \mathrm{~nm}$ coming from levels ${ }^{2} \mathrm{P}_{1 / 2},{ }^{4} \mathrm{G}_{7 / 2},{ }^{4} \mathrm{~F}_{7 / 2}$, and ${ }^{4} \mathrm{~F}_{3 / 2}$ respectively, are displayed in Fig. 5. The spectra show two bands, corresponding to the ${ }^{4} \mathrm{I}_{9 / 2} \rightarrow{ }^{4} \mathrm{~F}_{5 / 2},{ }^{4} \mathrm{~F}_{3 / 2}$ transitions. As can be observed, the spectra recorded by monitoring the luminescence at 595 and $760 \mathrm{~nm}$ are similar to each other, and do not show any significant differences with the excitation spectrum of the infrared emission. It must be mentioned that the spectra recorded at 535, 573, 655 and $668 \mathrm{~nm}$ are similar to the one recorded at $595 \mathrm{~nm}$. However, the spectrum recorded by monitoring the luminescence at $435 \mathrm{~nm}$ is slightly different, showing two additional weak peaks at 854 and $857 \mathrm{~nm}$ do not appear either in the excitation spectra of the other upconverted emissions nor in the excitation spectrum of the infrared emission or the one photon absorption spectrum. When pumping the sample at these wavelengths, the emission spectrum consists mainly of an intense peak at $435 \mathrm{~nm}$, originating in the ${ }^{2} \mathrm{P}_{1 / 2}$ level.

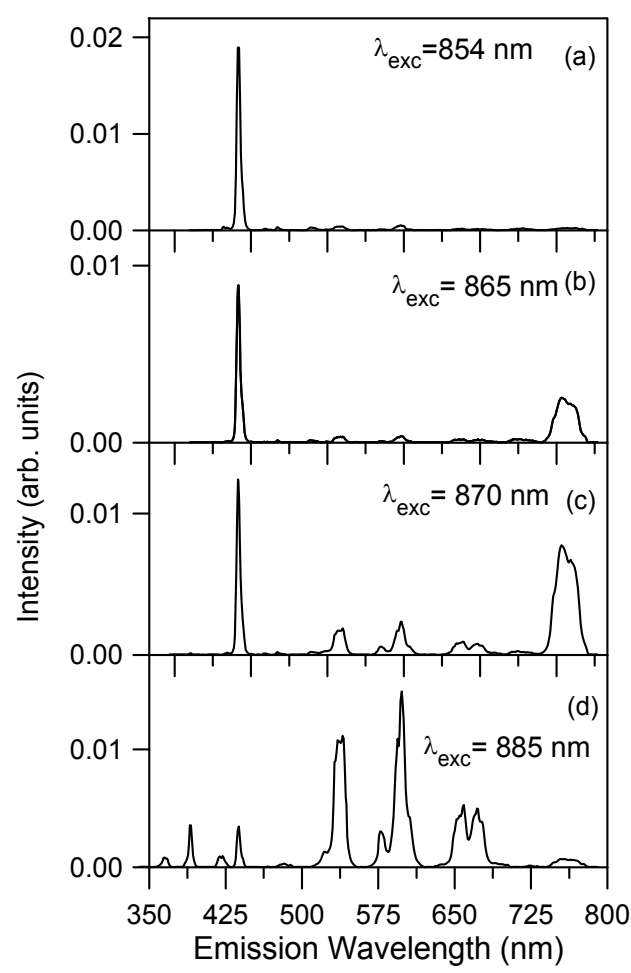

Fig. 4. Upconversion emission spectra obtained at different excitation wavelengths

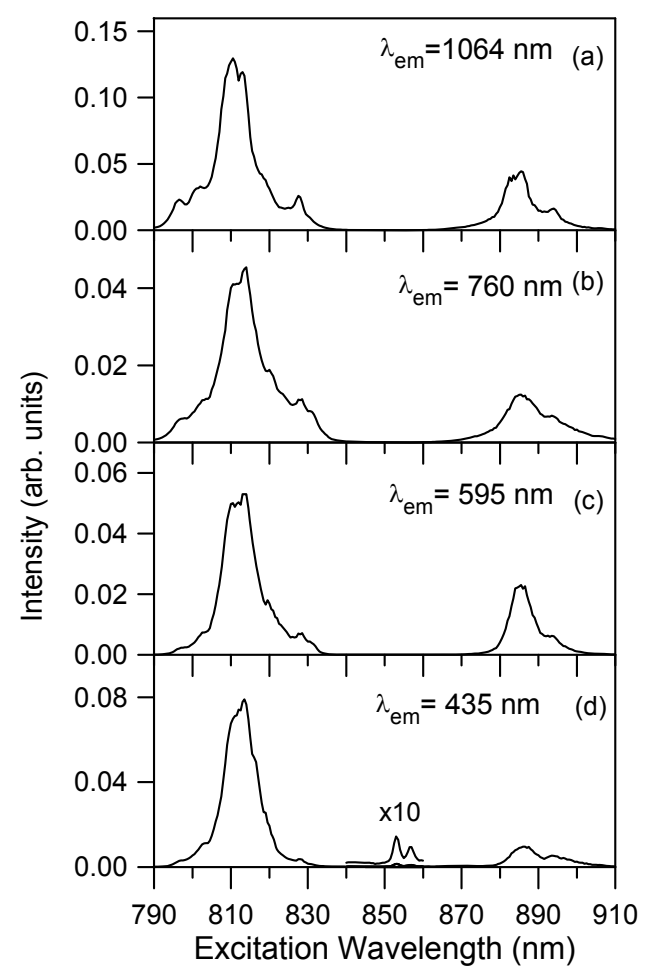

Fig. 5. Excitation spectra of the infrared luminescence and upconverted emissions

Concerning the emissions coming from levels ${ }^{4} \mathrm{G}_{7 / 2}-{ }^{4} \mathrm{G}_{9 / 2}$, the pump power dependence of the luminescence indicates that a two step process is responsible for these emissions. Moreover, the similarity between the excitation spectra and the one photon absorption spectrum as well as the decay of the upconverted luminescence showing longer time components than the natural lifetime of the emitting levels, point out to an ETU as the responsible mechanism for these emissions. These emitting levels can be populated through interaction of two ions that reach the ${ }^{4} \mathrm{~F}_{3 / 2}$ level after direct excitation of this level or after nonradiative decay from level ${ }^{4} \mathrm{~F}_{5 / 2}$. The ions in the ${ }^{4} \mathrm{~F}_{3 / 2}$ intermediate level can interact and reach the ${ }^{4} \mathrm{G}_{7 / 2}-{ }^{4} \mathrm{G}_{9 / 2}$ levels through transitions like $\left({ }^{4} \mathrm{~F}_{3 / 2}-{ }^{4} \mathrm{~F}_{3 / 2}\right)$ to $\left({ }^{4} \mathrm{G}_{7 / 2}-{ }^{4} \mathrm{I}_{13 / 2}\right)$ or $\left({ }^{4} \mathrm{~F}_{3 / 2}-{ }^{4} \mathrm{~F}_{3 / 2}\right)$ to $\left({ }^{2} \mathrm{G}_{9 / 2}-{ }^{4} \mathrm{I}_{11 / 2}\right)$ and subsequent decay to level ${ }^{4} \mathrm{G}_{7 / 2}$ (Fig. 6), and can reach the ${ }^{4} \mathrm{G}_{5 / 2}-{ }^{4} \mathrm{G}_{7 / 2}$ levels by $\left({ }^{4} \mathrm{~F}_{3 / 2}-{ }^{4} \mathrm{~F}_{3 / 2}\right)$ to $\left({ }^{4} \mathrm{G}_{5 / 2}\right.$ $\left.-{ }^{4} \mathrm{I}_{15 / 2}\right)$ transitions [10]. 
The red emission around $760 \mathrm{~nm}$, assigned to the ${ }^{4} \mathrm{~F}_{7 / 2} \rightarrow{ }^{4} \mathrm{I}_{9 / 2}$ transition, shows a quadratic dependence on pumping power and an excitation spectrum similar to the one photon absorption which indicates that an ETU process is responsible for this emission. Based on the energy levels diagram, energy transfer could take place by means of transitions $\left({ }^{4} \mathrm{I}_{11 / 2} \rightarrow{ }^{4} \mathrm{I}_{9 / 2}\right)$ and $\left({ }^{4} \mathrm{~F}_{3 / 2} \rightarrow{ }^{4} \mathrm{~F}_{7 / 2}\right)$. This process is non-resonant with an energy mismatch of $-50 \mathrm{~cm}^{-1}$.

The emission at $435 \mathrm{~nm}$ from level ${ }^{2} \mathrm{P}_{1 / 2}$ shows a quadratic dependence on pumping power which indicates that this level is populated by a two photon process. The experimental results indicate that the upconversion mechanism depends on the IR pumping wavelength. From the energy level diagram it can be seen that the ${ }^{2} \mathrm{P}_{1 / 2}$ level can be reached by absorption of two photons when exciting the sample into the ${ }^{4} \mathrm{~F}_{5 / 2}$ band: the first one excites electrons to the ${ }^{4} \mathrm{~F}_{5 / 2}$ level, then a non-radiative relaxation to the ${ }^{4} \mathrm{~F}_{3 / 2}$ level takes place, and a second IR photon is absorbed to reach the ${ }^{2} \mathrm{P}_{1 / 2}$ level. Still, the energy matching for this second transition is poor $\left(\Delta \mathrm{E} \approx 350 \mathrm{~cm}^{-1}\right)$. On the other hand, IR photons in resonance with the ${ }^{4} \mathrm{I}_{9 / 2} \rightarrow{ }^{4} \mathrm{~F}_{3 / 2}$ have not enough energy to allow electrons to reach the ${ }^{2} \mathrm{P}_{1 / 2}$ level, the energy mismatch being $\Delta \mathrm{E} \approx-400$ $\mathrm{cm}^{-1}$. The upconverted luminescence shows a two component decay with a long-lived component much longer than the natural lifetime of level ${ }^{2} \mathrm{P}_{1 / 2}$. This suggests that the main mechanism to populate level ${ }^{2} \mathrm{P}_{1 / 2}$ is ETU. As proposed in other chloride systems [11], a possible two step ETU process would involve two electrons in the ${ }^{4} \mathrm{~F}_{3 / 2}$ level, one of them being promoted to the ${ }^{2} \mathrm{P}_{1 / 2}$ level and the other one going down to the ground state. The additional energy needed to reach the ${ }^{2} \mathrm{P}_{1 / 2}$ level could be provided by the absorption of lattice phonons.

We already mentioned that, in addition to the peaks corresponding to resonant transitions from the ground state to the ${ }^{4} \mathrm{~F}_{3 / 2}$ and ${ }^{4} \mathrm{~F}_{5 / 2}$ levels, two new excitation peaks at 854 and $857 \mathrm{~nm}$ appear in the excitation spectrum of this emission, lying between the ${ }^{4} \mathrm{~F}_{3 / 2}$ and ${ }^{4} \mathrm{~F}_{5 / 2}$ bands. The energy of the photons at this wavelength is resonant with the ${ }^{4} \mathrm{~F}_{3 / 2} \rightarrow{ }^{2} \mathrm{P}_{1 / 2}$ transition and we have seen in Fig. 4 that excitation of the

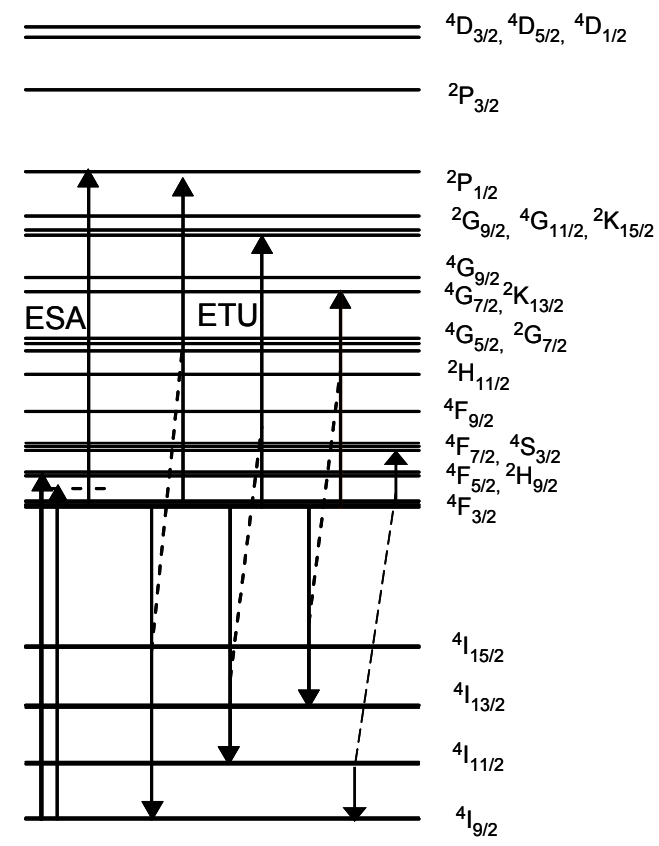

Fig. 6. Energy levels of $\mathrm{Nd}^{3+}$ in $\mathrm{KPC}$ crystal obtained from the room temperature absorption spectrum together with the proposed upconversion mechanisms.

sample at these wavelengths gives rise to emission from level ${ }^{2} \mathrm{P}_{1 / 2}$ mainly. Such excitation peaks have also been observed in other systems [12] and have been attributed to ${ }^{4} \mathrm{~F}_{3 / 2} \rightarrow{ }^{2} \mathrm{P}_{1 / 2}$ ESA processes from the low and high energy components of the ${ }^{4} \mathrm{~F}_{3 / 2}$ level, which is reached at its high energy side at these 
pumping wavelengths. The temporal behaviour of this upconverted luminescence, showing a lifetime very similar to the natural lifetime of level ${ }^{2} \mathrm{P}_{1 / 2}$ under direct excitation confirms the hypothesis of an ESA from level ${ }^{4} \mathrm{~F}_{3 / 2}$ as the responsible mechanism for the observed emission at $435 \mathrm{~nm}$ when pumping at 854 and $857 \mathrm{~nm}$. The upconversion mechanisms that we propose are depicted in Fig. 6.

\section{LASER COOLING}

\subsection{Photothermal quantum efficiency measurements}

The experimental set-up and procedure for the thermal deflection measurements has been described elsewhere [13]. The beam of a tunable ( $\lambda=780$ to $920 \mathrm{~nm}) \mathrm{cw}$ Ti-sapphire ring laser ( $8 \mathrm{GHz}$ bandwidth) with a maximum output power of $2.5 \mathrm{~W}$ entered the sample perpendicularly to the center of the face and it was modulated at low frequency $(1-10 \mathrm{~Hz})$ by a mechanical chopper. A fraction of the incident power was used for signal normalization. A copropagating helium-neon probe laser beam $(\lambda=632.8 \mathrm{~nm})$ was coaligned with the pump beam through a dichroic element. Both pump and probe copropagating beams were focused into the middle of the sample with diameters of $\sim 100 \mu \mathrm{m}$ and $\sim 60 \mu \mathrm{m}$ respectively. After leaving the sample, the beams passed through a second identical lens separated from the first one by a distance twice the focal length $(5 \mathrm{~cm})$ to avoid high divergence of the emerging beams. A second dichroic beam splitter deviated the pumping beam towards a pyroelectric detector that measured the transmitted pumping power. The probe beam passed through an interference filter before reaching a quadrant position detector to eliminate residual pumping radiation. The sample of size $4.5 \times 6.5 \times 2.7 \mathrm{~mm}^{3}$ was freely placed on a teflon holder inside a low vacuum $\left(\sim 10^{-2} \mathrm{mbar}\right)$ cryostat chamber at room temperature. Lock-in detection was used in the experiments. Thermal deflection waveforms were detected by using a digital scope.

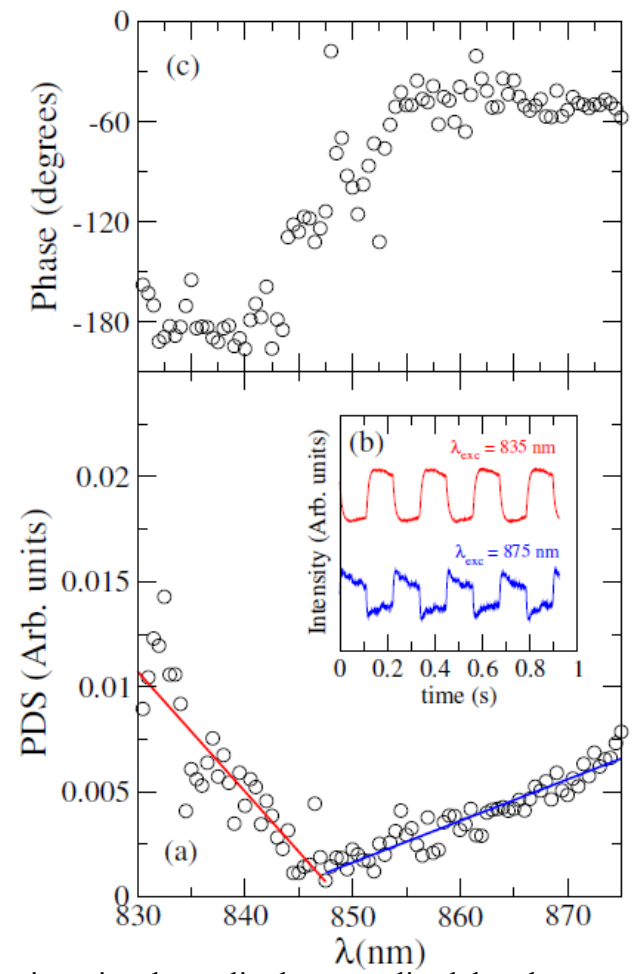

Fig. 7. (a) Photothermal deflection signal amplitude normalized by the sample absorption as a function of wavelength and (c) phase of the photothermal deflection signal for the $\mathrm{KPb}_{2} \mathrm{Cl}_{5}: \mathrm{Nd}^{3+}$ crystal. The inset shows the photothermal deflection signal waveforms in the heating $(835 \mathrm{~nm})$ and cooling $(875 \mathrm{~nm})$ regions for the $\mathrm{KPb}_{2} \mathrm{Cl}_{5}$ crystal at room temperature. 
The presence of internal cooling in this crystal was demonstrated by using the photothermal deflection technique. The cooling was obtained by exciting the $\mathrm{Nd}^{3+}$ ions between the ${ }^{4} \mathrm{~F}_{5 / 2}$ and ${ }^{4} \mathrm{~F}_{3 / 2}$ manifolds with a tunable Ti: sapphire laser. The cooling efficiency was evaluated at room temperature by measuring the quantum efficiency (QE) of the emission from the ${ }^{4} \mathrm{~F}_{3 / 2}$ manifold in the heating and cooling regions by means of the photothermal deflection spectroscopy in a collinear configuration [13].

The lock-in phase and amplitude of the photothermal deflection, normalized by the absorption, for the the $\mathrm{KPb}_{2} \mathrm{Cl}_{5}$ crystal, are displayed in Fig. 7 as a function of the pumping wavelength. As predicted by theory [14], a neat change of about $180^{\circ}$ can be observed during the transition from the heating to the cooling region at around $847 \mathrm{~nm}$ (Fig. 7(c)). The cooling efficiency estimated by using the QE measurements is about $0.82 \%$.

The room temperature photothermal deflection signal waveforms of $\mathrm{KPb}_{2} \mathrm{Cl}_{5}$ crystal recorded in the scope for different pumping wavelengths are shown in Fig. 7(b). The zero signal occurs around $847 \mathrm{~nm}$.

\subsection{Bulk cooling measurements}

The PDS results suggest that Nd-doped KPC is a good candidate for practical all-solid-state cooling applications. In order to assess this, we conducted measurements of the absolute temperature of a number of samples in both crystal and powder forms as a function of time under laser irradiation in the frequency range where we observed local internal cooling (between 847 and $875 \mathrm{~nm}$ ). To perform these measurements, a SILVER 480M (FLIR-CEDIP) infrared thermal camera with sensitivity of around 20 $\mathrm{mK}$ at $25^{\circ} \mathrm{C}$ was used. A refrigerated IsNb detector with a spatial resolution of 320 times 256 pixels is at the core of the infrared camera. Thermal scans at a rate of $5 \mathrm{~Hz}$ were acquired for time intervals ranging from 30 to 60 minutes. The camera was placed $12 \mathrm{~cm}$ apart from the window cryostat so that a lens with a field of vision of $25^{\circ}$ allowed focusing the camera on the sample. Some results obtained with this method were reported in [5]. Typically, the temperature of the sample initially rises till it reaches a stationary regime when the thermal load deposited on the material is compensated by the fluorescent losses. After this plateau, the temperature drops slightly. In the particular run depicted in Fig. 8, this drop is $0.25^{\circ} \mathrm{C}$. This result is quite impressive if one takes into account that we are using a single pass geometry, a very low power, and there is no other channel beyond the purely radiative one to evacuate the excess heat. Moreover, this result is even more striking if one takes into account what happens under the same irradiation conditions to an undoped KPC powder sample [5]: the temperature change does not saturate and, after a couple of minutes, the sample burns out. 


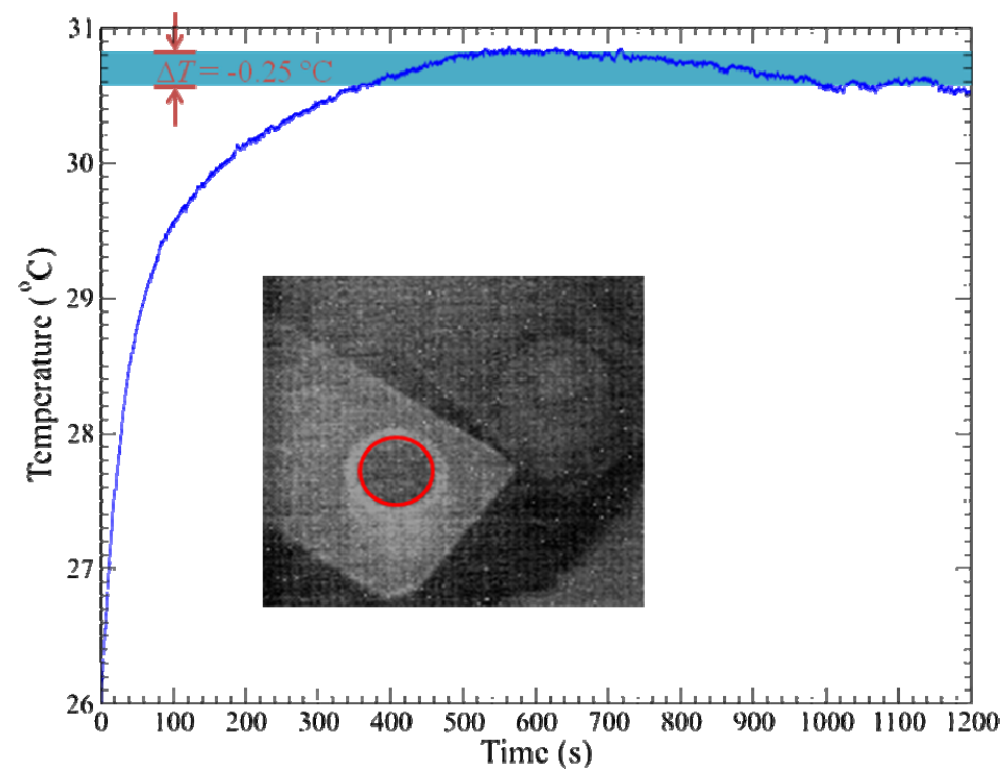

Fig. 8. Average temperature of a $1 \%$ Nd-doped KPC nanopowder as a function of time. The powder was irradiated with 1 watt of $870 \mathrm{~nm}$ laser light. The average temperature was calculated in the region delimited by the circle depicted in the inset. After 20 minutes, the temperature of the sample dropped $0.25^{\circ} \mathrm{C}$ with regards to the stationary value.

There is another remarkable fact about that result: as stated above, $870 \mathrm{~nm}$ is well inside the cooling region, as demonstrated by collinear PDS. However, a photon of $870 \mathrm{~nm}$ has an energy that is located above the barycentre of the emitting band $\left({ }^{4} \mathrm{~F}_{3 / 2}\right.$ band), according to the spectroscopic results in the previous section. Therefore, one would expect to obtain Stokes heating when measuring the average temperature of the sample, rather than the radiation balanced stationary regime shown in Fig. 8. The only way to reconcile the PDS, infrared imaging, and spectroscopic results is by assuming that, together with the conventional Stokes heating, there is an additional endothermic mechanism that offsets this heating and, at the same time, could explain the observed local internal cooling. Interestingly enough, the authors of the present manuscript made a theoretical proposal in reference 6 along these lines. We proposed that efficient infrared-to-visible upconversion processes occurring in Er-doped materials (mainly excited state absorption and energy transfer upconversion processes were considered in that work) could lead to an enhancement of the optical cooling efficiency and, were these processes efficient enough, to the occurrence of optical cooling above the barycentre of the emitting band. As explained in Section 3, the authors reported very efficient infrared-to-visible upconversion in Nd-doped KPC crystals, in the same spectral range as the one considered in the present work [10]. Therefore, everything seemed to indicate that infrared-to-visible upconversion could play an important role in these striking experimental results.

To further clarify the role of the infrared-to-visible upconversion processes in the observed cooling, we performed additional thermal imaging experiments where the wavelength of the laser was changed during the run. An example of such a run can be seen in Fig. 9 for a Nd-doped powder sample. For that particular experiment, we started pumping the sample with 0.5 watts of $870 \mathrm{~nm}$ laser light. As explained above, the temperature of the sample initially rises by about $4^{\circ} \mathrm{C}$, up to $29.4^{\circ} \mathrm{C}$, and then it reaches a plateau. We waited for about 30 minutes until the temperature of the sample was stable and then proceeded to change the wavelength to $875 \mathrm{~nm}$, almost outside the cooling region according to PDS measurements. Very quickly, the temperature of the sample rose by $0.7^{\circ} \mathrm{C}$, as expected. Interestingly, the upconverted blue emission intensity at this wavelength is very small. From this point on, we changed the wavelength in $5 \mathrm{~nm}$ intervals, down to $850 \mathrm{~nm}$ (see Fig. 10 for a detailed explanation). Each of these changes is accompanied by a noticeable temperature drop. In particular, when irradiated with $850 \mathrm{~nm}$ light, the temperature of the sample reaches a value of $27.9^{\circ} \mathrm{C}, 2.2^{\circ} \mathrm{C}$ below the maximum value of $30.1^{\circ} \mathrm{C}$ reached under $870 \mathrm{~nm}$ pumping. At that point, we changed the wavelength back to $870 \mathrm{~nm}$ to 


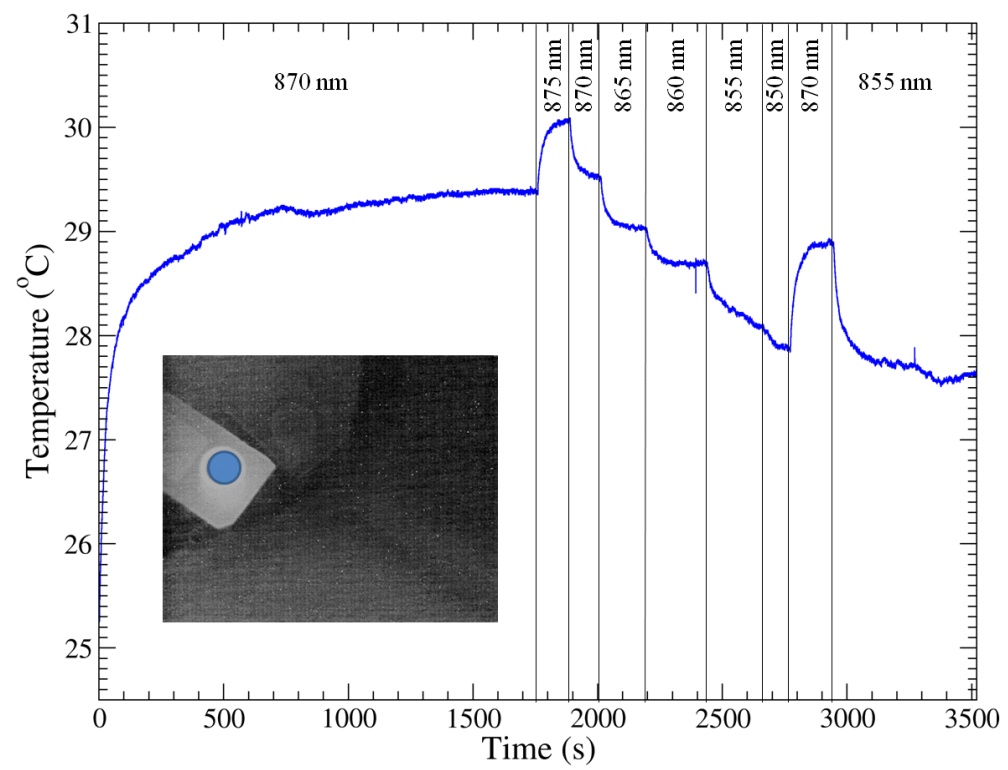

Fig. 9. Average temperature of a $1 \% \mathrm{Nd}$-doped KPC nanopowder as a function of time. The powder was irradiated with 0.5 watts of laser light with varying wavelength in the cooling region, as indicated in the figure. The average temperature was calculated in the region delimited by the circle depicted in the inset. After 60 minutes, the temperature of the sample dropped $1.9^{\circ} \mathrm{C}$ with regards to its stationary value reached at $870 \mathrm{~nm}$ pumping.

check the reproducibility of the results and, as expected, the temperature rapidly rose to $29.0^{\circ} \mathrm{C}$, very close to the stationary value of $29.4^{\circ} \mathrm{C}$ reached at the beginning of the run, showing that the material exhibits some, albeit small, thermal hysteresis. Changing back to $855 \mathrm{~nm}$, we reached the minimum temperature in this run of $27.5^{\circ} \mathrm{C}$. In brief, after a one hour run, we were able to attain a $1.9^{\circ} \mathrm{C}$ temperature drop in the powder sample with regards to the stationary temperature reached after 30 minutes irradiation at $870 \mathrm{~nm}$. We have depicted in Fig. 10 the minimum temperature attained at each wavelength during this run. This picture clearly shows that the temperature drop is related to the intensity of the upconverted blue emission (compare with Fig. 4). Indeed, the minimum temperature drop attained in this run occurs around $850-855 \mathrm{~nm}$, exactly where the blue upconverted emission intensity is maximum. Moreover, the excitation spectra also indicate that the blue upconverted emission originates around these wavelengths, that is, when one excites those phonon replicas of the ${ }^{4} \mathrm{~F}_{5 / 2}$ and ${ }^{4} \mathrm{~F}_{3 / 2}$ transitions.

We also wanted to test the theoretical proposal made by Ruan and Kaviany in 2006 [15]. These authors proposed that laser cooling in RE-doped nanocrystalline powder would be more efficient than in a crystal by a factor of almost 3! This is due to two main factors: firstly, multiple scattering in nanopowders can lead to light localization that increases the matter-light interaction and, secondly, the nanometric size of the powder grains modifies the phonon distribution in such a way that the long wavelength tail of this distribution is enhanced when compared with its crystalline counterpart. To test this idea, we conducted several runs in which samples of Nd-doped KPC in powder and crystal forms were irradiated simultaneously. Figure 11 is a typical example of such a run. There, we only show the portion of the run once the temperature of the samples reached the stationary plateau after 30 minutes irradiation at $870 \mathrm{~nm}$. At that point, we changed the wavelength to $855 \mathrm{~nm}$ and the temperature of the samples immediately started to drop. Around 800 seconds after changing the wavelength, we switched the laser off and on again to test the reproducibility of the temperature plateau and that lead to a temperature drop of 1.1 and $0.6^{\circ} \mathrm{C}$ for the powder and crystal, respectively, after a total run time of about 25 minutes, in qualitative agreement with the aforementioned idea first put forward by Ruan and Kaviany. 


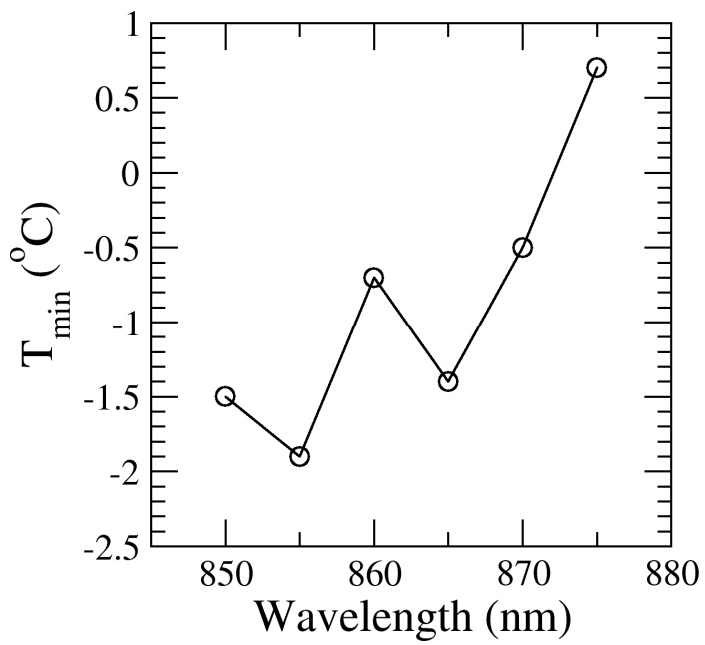

Fig. 10. Relative temperature change in the cooling region with regards to the stationary regime reached under pumping at $870 \mathrm{~nm}$ for the run depicted in Figure 10.

\subsection{Differential infrared thermography measurements}

All the above was explained in detail in Ref. [5]. However, one thing that is difficult to assess by using standard infrared thermography is the efficiency of the upconversion cooling channel. For this reason, we have developed a method that allows us to separate the upconversion contribution to cooling from the heating channels. We have termed this technique differential infrared thermography. Basically, we prepare two samples of the same host material (crystal or powder), one doped with $\mathrm{Nd}^{3+}$ and one

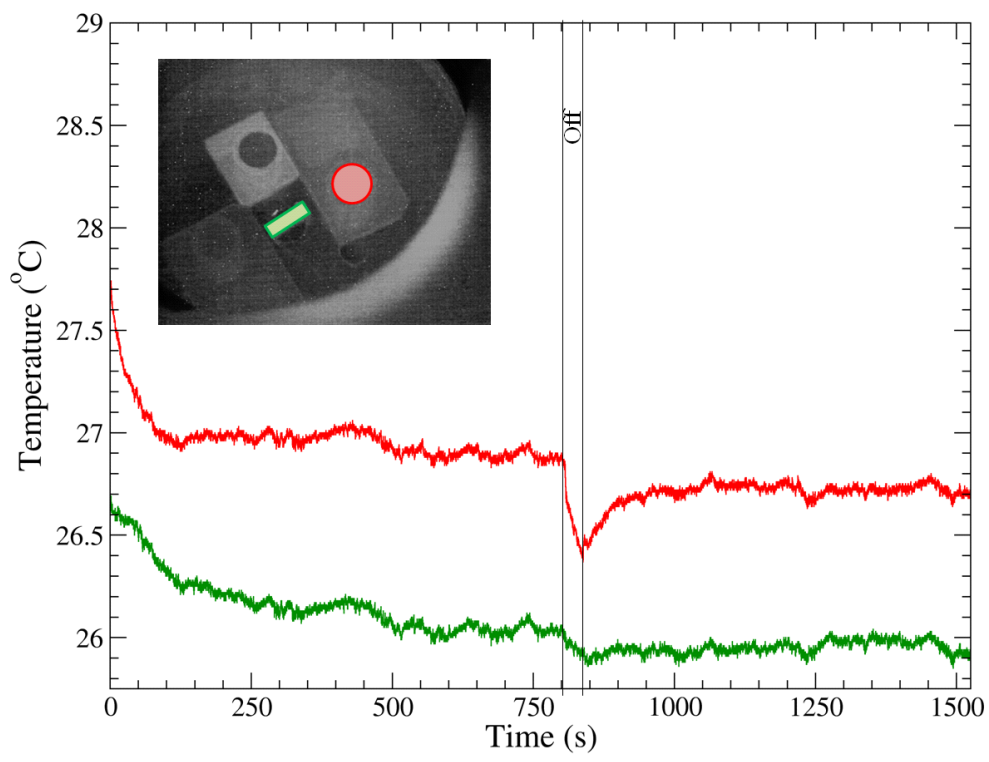

Fig. 11. Average temperature of a $1 \%$ Nd-doped KPC nanopowder and crystal as a function of time. The powder was irradiated with 0.5 watts of $855 \mathrm{~nm}$ laser light. The average temperature was calculated in the region delimited by the circle depicted in the inset. After 800 seconds, the laser was switched off and on again. The crystal was simultaneously irradiated with 1.2 watts of $855 \mathrm{~nm}$ laser light. The average temperature was calculated in the region delimited by the rectangle depicted in the inset. After 25 minutes, the temperature of the powder dropped $1.1^{\circ} \mathrm{C}$ with regards to its initial, stationary value reached at $870 \mathrm{~nm}$ pumping, whereas the temperature of the crystal dropped by $0.6^{\circ} \mathrm{C}$. 
undoped and they are located side by side inside the cryostat (for the powder samples we use a especial cylindrical holder) as shown in Figs. 12a and 13a. Both samples are irradiated simultaneously taking especial care of distributing the laser spot equally among them. In this way, we can use the non-doped sample as a reference and subtract any contributions to heating not related to processes involving the optically active ions, such as background absorption processes, power fluctuations, etc.

A first example of the application of this technique to the study of optical cooling in a sample of $\mathrm{KPb}_{2} \mathrm{Cl}_{5}$ crystal doped with $1 \% \mathrm{Nd}^{3+}$ can be seen in Figure 12. There, the temperature dynamics of both a doped sample together with a similar, undoped one, are shown. For comparison, the evolution of the temperature at the laser spot and the average temperature of both samples are displayed. For that particular experimental run, the samples were pumped with 1.5 watts of laser light at $850 \mathrm{~nm}$, just above the lower boundary of the spectral range where internal optical cooling was observed. It is difficult to extract any clear information from the absolute temperatures. Initially, the temperature of both samples rises quite steeply before the growth rate becomes more gradual. After that, if one waits long enough, a plateau is usually reached and a later temperature drop occurs, as explained before and as can also be seen in Fig. 8. For the particular run depicted in Fig. 12, however, we turned the laser off before reaching that plateau, around 20 minutes after irradiation started. If we now subtract the temperature curve of the undoped sample to the temperature from the doped one, as shown in Fig. 12c, it is easy to notice that the temperature at the laser spot in the doped sample is consistently $0.25^{\circ} \mathrm{C}$ below that of the undoped one, independently of the thermal load in the cavity, power variations or any other noise sources, which is an indication of, at least, reduced heating in the doped crystal. If we turn to the average temperature, however, we can see that the corresponding difference is almost zero, indicating that, strictly speaking, no bulk cooling is occurring.

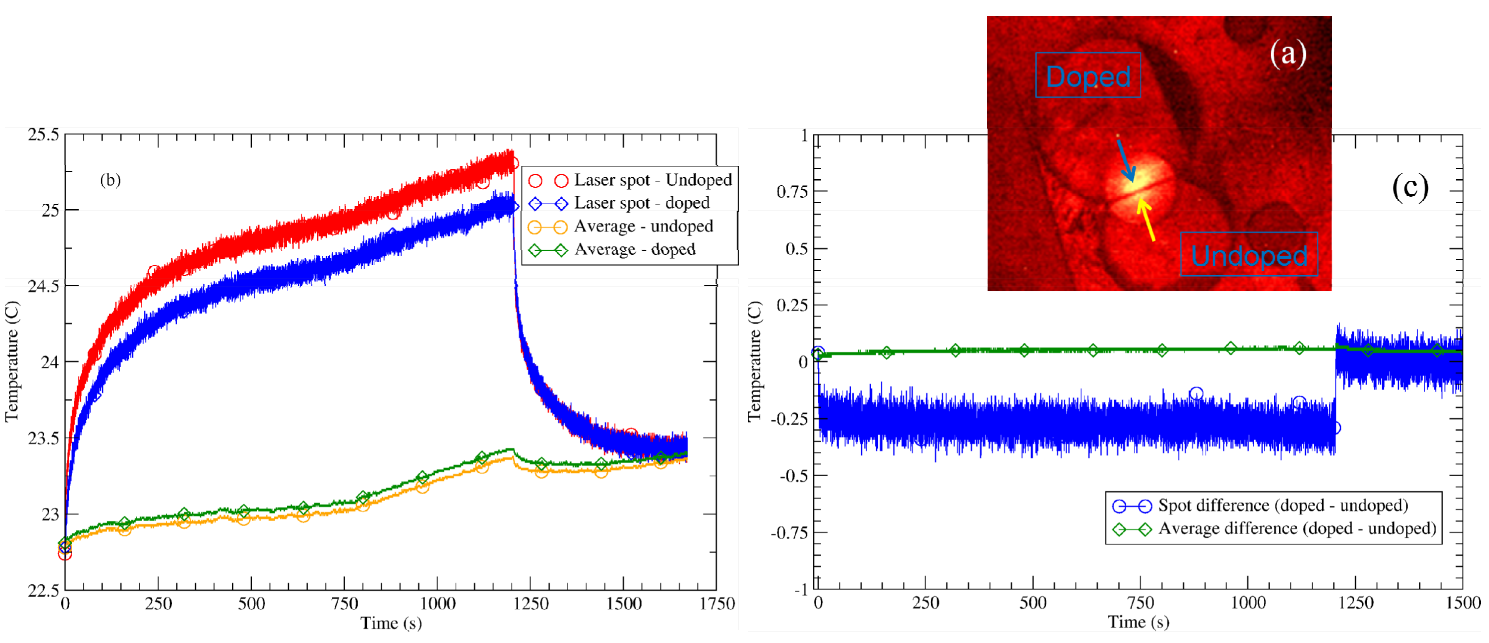

Figure 12. (a) Sample arrangement as seen by infrared thermography. A piece of undoped crystal is set side by side with a doped piece of the same host matrix. (b) Temperature dynamics at the laser spots indicated in (a) and average temperature of the samples. (c) Temperature differences: the temperature of the undoped sample is subtracted from the doped one.

The situation, however, is quite different for Nd-doped KPC powder samples, as the temperature differences are more noticeable for the reasons explained in Ref. [14]. In Fig. 13, we investigated the region below the barycenter of the ${ }^{4} \mathrm{I}_{3 / 2}$ emitting band, where, together with the upconversion processes described above, one expects standard anti-Stokes absorption processes to occur. For this type of measurements, one half of a cylindrical shaped holder was filled with the doped powder whereas the other half was filled with the undoped material. After reaching the stationary regime using $855 \mathrm{~nm}$ laser light, we changed the wavelength to $900 \mathrm{~nm}$, at the barycenter of the emitting band, with a pump power of $430 \mathrm{~mW}$. Immediately, the average temperature of the doped sample started to rise faster than the undoped one. This is to be expected, as the absorption is larger for the doped material (ideally the 
undoped material is transparent in this spectral range, albeit a small background absorption is always present in real materials [2]) and neither anti-Stokes processes nor upconversion ones occur. Then we changed the wavelength of the laser to $910 \mathrm{~nm}$, in resonance with a one-phonon annihilation assisted process, and the temperature of both materials drop at this wavelength, even though the background absorption in the undoped material should be frequency independent. However, this temperature change is not due to a change in the absorption of the material, but rather to the fact that the laser power changes from $430 \mathrm{~mW}$ to $405 \mathrm{~mW}$ at this wavelength. If we then change the wavelength to $918 \mathrm{~nm}$, in resonance with a two-phonon annihilation process and the various endothermic upconversion processes described in the previous section, there is a reversal of the average temperature: the average temperature of the doped sample drops below the average temperature of the undoped one, indicating that cooling occurs. Again, there is a global temperature drop due to a change in the laser power from $405 \mathrm{~mW}$ to $380 \mathrm{~mW}$. At that point, we switched off the laser and changed its output power to $600 \mathrm{~mW}$. Immediately, the global temperature of the system starts to rise, but it is clear that the temperature difference between the doped and undoped samples is larger. It is even larger if the power is increased to $800 \mathrm{~mW}$. Unfortunately, at this power level, the undoped sample burned up after a few minutes.

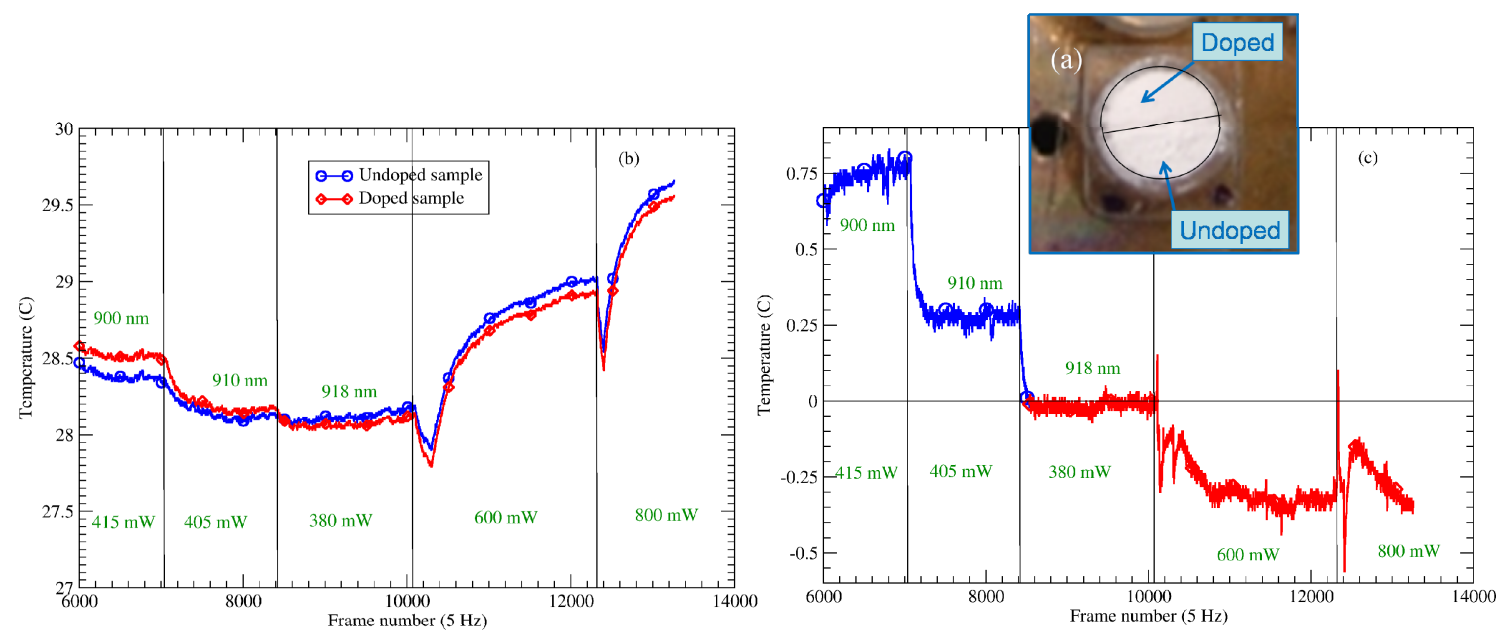

Figure 13. (a) Powder samples arrangement in the cylindrical holder.. (b) Average temperature dynamics after reaching the stationary regime at 900,910 , and $910 \mathrm{~nm}$. The pumping power is also indicated (c) Differential thermograph as a function of time.

If we now look to the differential thermograph in Fig. 13c , the facts explained in the previous paragraph are even more evident. There is a neat transition from heating to cooling at $918 \mathrm{~nm}$, in exact resonance with a two-phonon process, even at a pumping power as low as $380 \mathrm{~mW}$ ! Moreover, the cooling efficiency is larger the larger the pumping power. Unfortunately, the burning temperature of the undoped sample limits the maximum pumping power one can reach. To try and remediate this limitation, we used a divergent lens to expand the laser beam, so that the intensity was smaller for the same pumping power. In this way, we were able to reach pumping powers as large as $1250 \mathrm{~mW}$ without burning the undoped portion of the sample. As shown in Fig. 14, the higher the power, the more efficient the cooling in the doped material. Indeed, at $1250 \mathrm{~mW}$ pumping power we achieved an average temperature difference of about $-0.65^{\circ} \mathrm{C}$. 


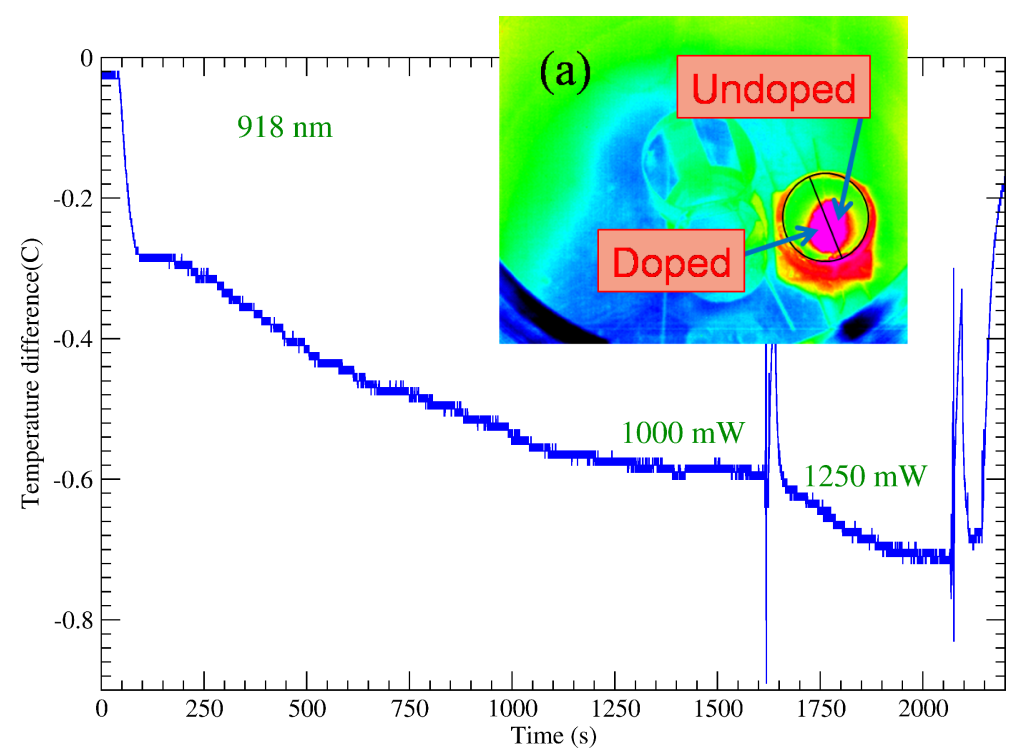

Figure 14. Differential thermograph of a powder sample at $918 \mathrm{~nm}$. A divergent lens was used to expand the laser beam (the big stain in between both the doped and undoped samples). Pumping powers are indicated.

\section{CONCLUSIONS}

In summary, we have reported a comprehensive review of our investigations on optical cooling of Nddoped KPC nanopowders and crystals. These solids clearly exhibit local internal cooling as probed by collinear photothermal deflection spectroscopy. Bulk cooling measurements based on infrared thermography show that the cooling processes occurring in these low-energy-phonon materials are nonstandard and, in fact, cooling is assisted by efficient infrared-to-visible upconversion processes. These have been exhaustively analyzed from the spectroscopic point of view and one reaches the conclusion that this type of cooling pathway can lead to optical cooling even above the barycentre of the emitting band, in contrast with conventional anti-Stokes absorption processes.

To further delve into the nature of the unconventional cooling occurring in these materials, we used the Differential Infrared Thermography technique. This method allows us to subtract the effects of background absorption, noise sources, and power fluctuations. By using this technique, it is shown that infrared-to-visible upconversion can completely offset the heat load deposited in the doped material so that its temperature remains constant or even decreases by almost $0.7^{\circ} \mathrm{C}$ with regards to the temperature at the stationary regime in the Nd-doped material in powder form. Furthermore, below the barycentre of the emitting band, conventional anti-Stokes cooling occurs at exact resonance with a two-phonon absorption assisted process.

All this body of results clearly demonstrate that very pure low-energy-phonon materials dopen with $\mathrm{Nd}$ are very promising candidates for the development of optical cryocoolers and radiation-balanced lasers.

\section{ACKNOWLEDGMENTS}

This work was supported by the Spanish Government MEC under projects FIS2011-27968, Consolider SAUUL CSD2007-00013, and Basque Country Government (IT-331-07). Daniel Sola thanks the JAEDOC program and the Science and Technology Inter-Ministry commission of Spain and FEDER founds of the EC under project MAT2009-13979-C03-03 for the financial support of his contract. 


\section{REFERENCES}

[1] R. I. Epstein, M. I. Buchwald, B. C. Edwards, T. R. Gosnell, and C. E. Mungan, "Observation of laser-induced fluorescent cooling of a solid", Nature 377, pp. 500-503, 1995.

[2] C.W. Hoyt, M.P. Hasselbeck, M. Sheik-Bahae, R.I. Epstein, S. Greenfield, J. Thiede, J. Distel, and J. Valencia, "Advances in laser cooling of thulium-doped glass", J. Opt. Soc. Am. B 20, pp. 1066-1074, 2003 and references therein.

[3] M. Sheik-Bahae and R. I. Epstein, "Optical Refrigeration”, Nature Photonics 1, pp. 693-699, 2007. See also Denis V. Seletskiy, Seth D. Melgaard, Stefano Bigotta, Alberto Di Lieto, Mauro Tonelli, Mansoor Sheik-Bahae, "Laser cooling of solids to cryogenic temperatures", Nature Photonics, 2010.

[4] J. Fernández, A.J. García-Adeva, and R. Balda, "Anti-Stokes Laser Cooling in Bulk Erbium-Doped Materials", Phys. Rev. Lett. 97, pp. 033001-4, 2006.

[5] A.J. Garcia-Adeva, R. Balda, M. Al Saleh, and J. Fernandez, "Local internal and bulk optical cooling in Nd-doped crystals and nanocrystalline powders", Proc. of the SPIE 7614, 76140A (2010).

[6] A.J. Garcia-Adeva, R. Balda, J. Fernández, "Upconversion cooling of Er-doped low-phonon fluorescent solids", Phys. Rev. B 79, 033110, 2009.

[7] R. Balda, A. J. Garcia-Adeva, M. Voda, and J. Fernández, "Upconversion processes in $\mathrm{Er}^{3+}$-doped $\mathrm{KPb}_{2} \mathrm{Cl}_{5}$ ", Phys. Rev. B 69, pp. 2052031-2052038, 2004.

[8] M.C. Nostrand, R.H. Page, S.A. Payne, L.I. Isaenko, and A.P. Yelisseyev, "Optical properties of $\mathrm{Dy}^{3+}$-and $\mathrm{Nd}^{3+}$-doped $\mathrm{KPb}_{2} \mathrm{Cl}_{5}$ ", J. Opt. Soc. Am. B 18, pp. 264-276, 2001.

[9] A. Mendioroz, J. Fernández, M. Voda, M. Al-Saleh, R. Balda, and A.J. Garcia-Adeva, "Anti-Stokes laser cooling in $\mathrm{Yb}^{3+}$-doped $\mathrm{KPb}_{2} \mathrm{Cl}_{5}$ crystal", Opt. Lett. 27, pp. 1525-1527, 2002.

[10] A. Mendioroz, R. Balda, M. Voda, M. Al-Saleh, J. Fernández, "Infrared to visible and ultraviolet upconversion processes in $\mathrm{Nd}^{3+}$-doped potassium lead chloride crystal", Opt. Mater. 26, pp. 351-357, 2004.

[11] T. Tsuneoka, K. Kojima, S. Bojja, "Upconversion fluorescence and low temperature fluorescence properties in $\mathrm{Nd}^{3+}$-doped $\mathrm{ZnCl}_{2}$-based glass", J. Non-Cryst. Solids 202, pp. 297-302, 1996.

[12] R. Balda, M. Sanz, A. Mendioroz, J. Fernández, L.S. Griscom, J.L. Adam, "Infrared-to-visible upconversion in Nd-doped chalcohalide glasses", Phys. Rev. B 64, 144101, 2001.

[13] J. Fernández, A. Mendioroz, A.J. García, R. Balda, J.L. Adam, "Laser-induced internal cooling of $\mathrm{Yb}^{3+}$-doped fluoride-based glasses", J. of Alloys and Compounds 323-324, pp. 239-244, 2001.

[14] X.L. Ruan and M. Kaviany, "Enhanced laser cooling of rare-earth-ion-doped nanocrystalline powders", Phys. Rev. B 73, APR 2006. 